\title{
Use of the Heartrail ST01 catheter for optimized aspiration thrombectomy in a patient with ST-segment elevation myocardial infarction with a large intracoronary thrombus
}

\author{
Haibo Chen, Lingjun Zhu, Jun Jiang \\ Department of Cardiology, Second Affiliated Hospital, Zhejiang University School of Medicine, Hangzhou, China
}

Adv Interv Cardiol 2020; 16, 4 (62): 509-511

DOI: https://doi.org/10.5114/aic.2020.101780

A 91-year-old woman presented with ST-segment elevation myocardial infarction (STEMI) after taking a meal. The emergent coronary angiography demonstrated acute proximal occlusion of the right coronary artery (RCA) (Figure $1 \mathrm{~A}$ ). Initial aspiration thrombectomy (AT) with a Thrombuster II (Kaneka Medical) thrombus aspiration catheter (extraction area (EA) $0.95 \mathrm{~mm}^{2}$ ) recovered TIMI III flow. However, post-aspiration angiography demonstrated severe residual thrombus burden (Figure 1 B). Because of its large suction area, a guide extension mother-and-child catheter, Heartrail ST01 $5 \mathrm{Fr}$ (cross section area, CSA $1.77 \mathrm{~mm}^{2}$, Terumo Medical) catheter was positioned proximal to the site of the occlusion (Figure $1 \mathrm{C}$ ). Aspiration was performed with suction pressure generated by a $30 \mathrm{ml}$ vacuum syringe and a larger and long embolus was sucked out (Figure $1 \mathrm{E})$, resulting in evident reduction of thrombus burden (Figure $1 \mathrm{D}$ ) and it was completed with a $3.5 \times 30 \mathrm{~mm}$ zotarolimus-eluting stent (Endeavor, Medtronic) implantation. Histological examination showed thrombus with abundant infiltration of neutrophil (Figure $1 \mathrm{~F}$ ). Furthermore, the patient received $100 \mathrm{mg}$ of aspirin once daily and $90 \mathrm{mg}$ of ticagrelor twice daily for 2 weeks, which was replaced by $100 \mathrm{mg}$ of aspirin plus $75 \mathrm{mg}$ of clopidogrel once daily with an 8-month follow-up to date and the patient has not suffered cardiovascular or bleeding events.

Despite improved clinical outcomes observed in early trials, recent randomized trials demonstrated that, in STEMI patients, as compared with percutaneous coronary intervention $(\mathrm{PCl})$ alone, routine manual thrombectomy followed by primary $\mathrm{PCI}(\mathrm{PPCl})$ had no advantages in reduction of all-cause mortality, cardiovascular death, recurrent myocardial infarction, cardiogenic shock, or NYHA class IV heart failure but was associated with an increased rate of stroke [1]. Thus, routine thrombus aspiration is not recommended, but bailout in certain cases may be considered [2].

The success of manual aspiration is limited by multiple factors including catheter tip EA, vacuum generation, deliverability, and vessel and thrombus characteristics. A majority of PPCls are performed through 6 Fr systems, and the greatest shortcoming is their small inner CSA (0.80 to $1.24 \mathrm{~mm}^{2}$ ). Moreover, utilizing $6 \mathrm{Fr}$ guide systems markedly impeded the use of larger AT devices. Therefore, optimized AT with guide extension catheters seems to be a reasonable choice. In previous studies, guide extension catheters have been reported to have applications in facilitating equipment delivery, providing vessel support, thrombus aspiration, retrieval of lost devices, selective vessel contrast injection, and facilitation of chronic total occlusion lesion management [3]. Furthermore, various guide extension catheters, such as the same series Heartrail II-ST01 [4], GuideLiner V2 (EA $1.58 \mathrm{~mm}^{2}$, Vascular Solutions) and Guidezilla (EA $1.65 \mathrm{~mm}^{2}$, Boston Scientific), have been reported for AT, showing their outstanding efficacy in AT and safety for distal coronary embolization as well as stroke. The Heartrail ST01 catheter is a $120 \mathrm{~cm}$ straight tip $5 \mathrm{Fr}$ with a CSA $1.77 \mathrm{~mm}^{2}$ resulting in an evidently larger aspiration force. It is the first time to apply the Heartrail ST01 to successfully extract such a large and long thrombus (approximate length $=56.8 \mathrm{~mm}$, referring to the outer diameter of the filter) in the RCA, demonstrating the feasibility of utilization of the guide extension catheter for AT during PPCI with severe thrombus burden. 

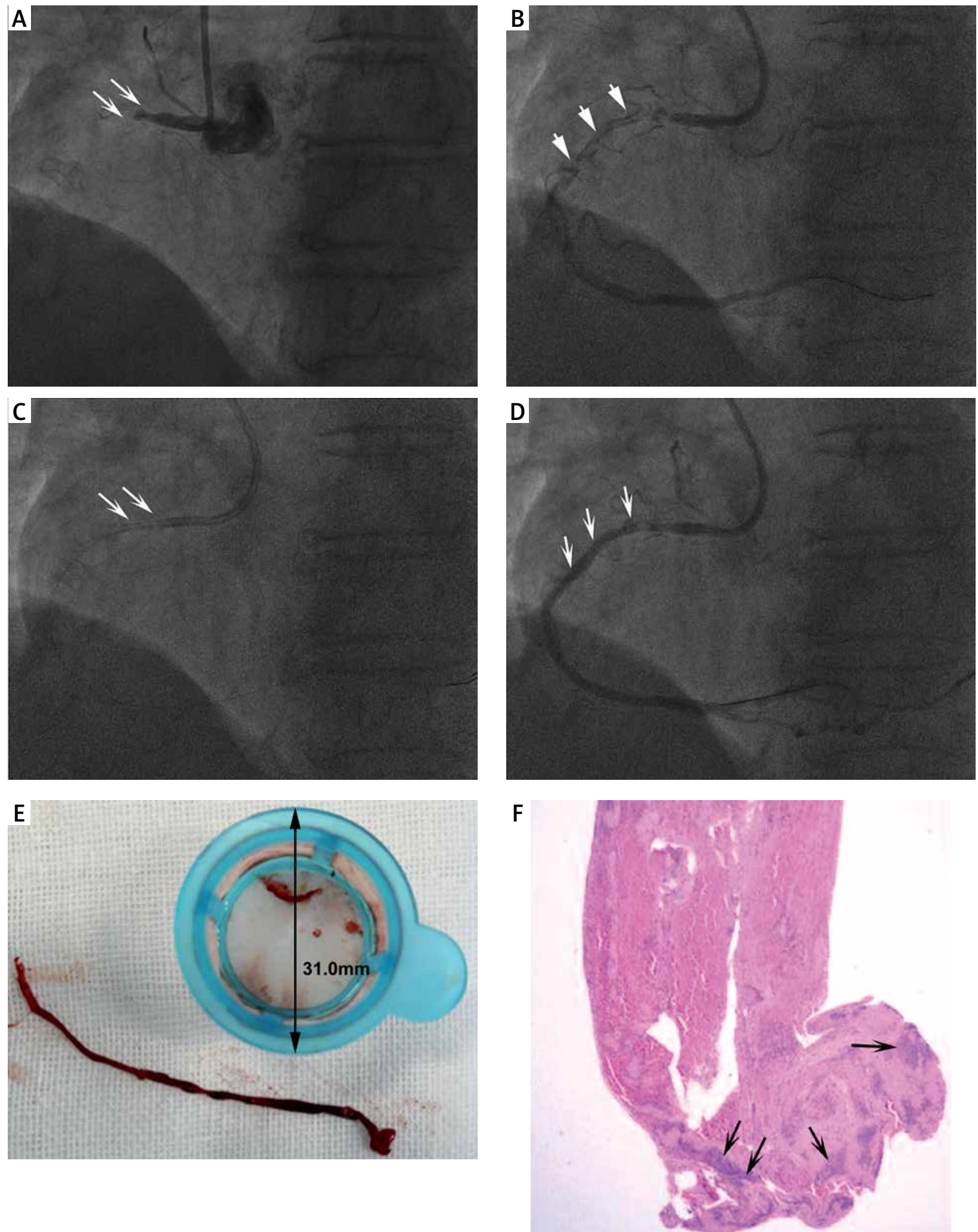

Figure 1. A - Emergent angiography, proximal occlusion of RCA (white arrow). B - Severe residual thrombus burden after initial aspiration thrombectomy with Thrombuster II catheter (white arrowhead). C - Optimized aspiration thrombectomy with Heartrail ST01 guide extension catheter (white arrow). D - Evident reduction of thrombus burden after optimized aspiration thrombectomy (white arrow). E - A large and long thrombus extracted with guide extension catheter. F - Histopathological result of extracted thrombus, infiltrated with abundant neutrophil (black arrow) 


\section{Acknowledgments}

This work was supported by a grant from the National Natural Science Foundation of China (No. 81700391).

\section{Conflict of interest}

The authors declare no conflict of interest.

\section{References}

1. Jolly SS, Cairns JA, Yusuf S, et al. Randomized trial of primary PCI with or without routine manual thrombectomy. N Engl J Med 2015; 372: 1389-98.

2. Ibanez B, James S, Agewall S, et al. 2017 ESC Guidelines for the management of acute myocardial infarction in patients presenting with ST-segment elevation: The Task Force for the management of acute myocardial infarction in patients presenting with ST-segment elevation of the European Society of Cardiology (ESC). Eur Heart J 2018; 39: 119-77.

3. Duong T, Christopoulos G, Luna M, et al. Frequency, indications, and outcomes of guide catheter extension use in percutaneous coronary intervention. J Invasive Cardiol 2015; 27: E211-5.

4. Ciecwierz D, Mielczarek $M$, Jaguszewski $M$, et al. The first reported aspiration thrombectomy with a guide extension mother-and-child catheter in ST elevation myocardial infarction due to bacterial vegetation coronary artery embolism. Adv Interv Cardiol 2016; 12: 70-2. 